\title{
Influence of an extended non-equilibrium region on the far-field of grid turbulence
}

\author{
R. J. Hearst and P. Lavoie
}

\begin{abstract}
The turbulence produced by two regular square mesh grids is compared to that produced by a square-fractal-element grid composed of an array of small square fractals. All three grids have approximately the same blockage. One of the regular grids is designed to have the same mesh length, $M$, as the fractal element grid, while the other matches the maximum bar thickness of the fractal. The transition of the turbulence from a non-equilibrium to a near equilibrium regime is assessed through the scale-by-scale kinetic energy budget and the velocity derivative skewness. It is found that the turbulence produced by all three grids agrees with many of the predictions for equilibrium phenomenology after approximately $20 M$, with the regular grids reaching quasi-equilibrium earlier than the fractal. In the far-field, the fractal grid produces comparable or lower $R e_{\lambda}$ than the regular grids in both dimensional and non-dimensional measurements of the streamwise position. This is attributed to an extended rapidly decaying non-equilibrium region in the wake of the fractal grid relative to the regular grids.
\end{abstract}

\section{Introduction}

Recent grid turbulence experiments have focussed on the 'non-equilibrium' region that is produced immediately downstream of the grid. Interest in this area was garnered by the studies of Vassilicos and co-workers in the wake of space-filling square

R. Jason Hearst

Institute for Aerospace Studies, University of Toronto, M3H 5T6, Canada

Presently at: Engineering and the Environment, University of Southampton, SO17 1BJ, United

Kingdom

e-mail: r.j.hearst@southampton.ac.uk

Philippe Lavoie

Institute for Aerospace Studies, University of Toronto, M3H 5T6, Canada

e-mail: lavoie@utias.utoronto.ca 
fractal grids, e.g., $[5,10,15,18]$. A space-filling square fractal is a single square fractal pattern that occupies the entire wind tunnel cross-section. Recently, observations of non-equilibrium turbulence have also been reported in the wake of an array of different grids, including: multi-scale cross grids [13], fractal element grids $[7,8,20]$, and regular grids [11, 19]. It has also been demonstrated that for all these grid geometries the non-equilibrium turbulence evolves into one that better approximates equilibrium turbulence ${ }^{1}$ sufficiently far downstream $[7,8,11,12,13,22]$.

Observations of quasi-equilibrium turbulence in the wake of grids has been characterized by a homogeneous field with an energy decay rate in the range $-1 \gtrsim n \gtrsim-1.4$, for a power-law decay of the form $\left\langle u^{2}\right\rangle \sim\left(x-x_{0}\right)^{n}$, and constant normalized dissipation scaling, $C_{\varepsilon} \sim \varepsilon L / u^{\prime 3}$, where $\varepsilon$ is the dissipation rate of turbulent kinetic energy and $L$ is the integral length scale. Non-equilibrium turbulence contrasts on all three of these points. The transverse flow fields experience non-zero transverse transport of turbulent kinetic energy and production, the energy decays at an accelerated rate, $n \lesssim-2$, and $C_{\varepsilon}$ grows rapidly $[7,8,16,18,20]$.

The transition between these regions has been characterized in several ways. Isaza et al. [11] recalled from Batchelor [1] that for equilibrium turbulence, the velocity derivative skewness,

$$
S(\partial u / \partial x)=\frac{\left\langle(\partial u / \partial x)^{3}\right\rangle}{\left\langle(\partial u / \partial x)^{2}\right\rangle^{3 / 2}},
$$

should be constant, and hence areas where $S(\partial u / \partial x)$ varied were associated with non-equilibrium turbulence. Hearst and Lavoie [7] discriminated between the regions by identifying where the flow became homogeneous in transverse planes and noting this coincided with a change in the decay rate, $n$. Later, these same authors used the scale-by-scale kinetic energy budget to determine the difference between the flow regions [8]. The scale-by-scale kinetic energy budget for grid turbulence is given by [4],

$$
-\left\langle(\delta u)(\delta q)^{2}\right\rangle+2 v \frac{\mathrm{d}}{\mathrm{d} r}\left\langle(\delta q)^{2}\right\rangle-\frac{U}{r^{2}} \int_{0}^{r} s \frac{\partial}{\partial x}\left\langle(\delta q)^{2}\right\rangle \mathrm{d} s=\frac{4}{3}\langle\varepsilon\rangle r
$$

where $\delta \alpha=\alpha(x+r)-\alpha(x),\left\langle(\delta q)^{2}\right\rangle=\left\langle(\delta u)^{2}\right\rangle+\left\langle(\delta v)^{2}\right\rangle+\left\langle(\delta w)^{2}\right\rangle,\left\langle(\delta u)(\delta q)^{2}\right\rangle=$ $\left\langle(\delta u)^{3}\right\rangle+\left\langle(\delta u)(\delta v)^{2}\right\rangle+\left\langle(\delta u)(\delta w)^{2}\right\rangle$, and $s$ is a dummy integration variable. Equation (2) may be represented simply as $G+D+I=C$. $G$ represents energy transfer through advection. $D$ represents the energy transfer through molecular diffusion. $I$ accounts for the influence of longitudinal inhomogeneity resulting from the decay of turbulence behind the grid. Finally, $C$ represents dissipation. Equation (2) is satisfied for equilibrium turbulence but not for non-equilibrium turbulence due to the presence of production and transverse transport [8]. Hence, assessing the validity

\footnotetext{
${ }^{1}$ One of the most oft cited distinctions between true Richardson-Kolmogorov equilibrium phenomenology and the 'quasi-equilibrium' turbulence measured in the far-field of regular grids has been a lack of a $k^{-5 / 3}$ velocity spectrum.
} 
of (2) at each location in the flow can distinguish regions that approximate the two phenomenologies.

It would appear that one of the primary benefits of the fractal geometry is that it creates a physically large region of non-equilibrium turbulence relative to other grid geometries [7]. As such, the characteristically high $\operatorname{Re}_{\lambda}=\left\langle u^{2}\right\rangle^{1 / 2} \lambda / v$ region close to the grid extends over a significant downstream range. However, there has not been a detailed investigation of the far-field repercussions of extending the near-grid non-equilibrium region. Here, we first investigate the scale-by-scale energy budget and $S(\partial u / \partial x)$ to discriminate the non-equilibrium and quasi-equilibrium regions. We then compare two regular grids with a fractal element grid at the same $R e_{M}=$ $U_{0} M / v$, where $U_{0}$ is the velocity immediately upstream of the grid, to identify how extending the non-equilibrium region influences the relative magnitude of $R e_{\lambda}$ in the far-field.

\section{Experimental details}

Three different passive grids were investigated for the present study. The first was the square-fractal-element grid used in [7, 8], which is referred to here as $F s 39$. This grid consists of a $12 \times 8$ array of 3 fractal iteration square fractal elements mounted to a background mesh with $M=100 \mathrm{~mm}$, and thickness $\tau_{0}=6.7 \mathrm{~mm}$. Fs39 has $\sigma=0.39$ blockage, and is described in more detail in [7, 8]. A regular square mesh grid, $S q 39$, with $M=100 \mathrm{~mm}, \tau_{0}=22.0 \mathrm{~mm}$, and $\sigma=0.39$, was deliberately designed to match $M$ and $\sigma$ to $F$ s39. A second regular grid, $R d 38$, with $M=32 \mathrm{~mm}, \tau_{0}=6.8 \mathrm{~mm}$, and $\sigma=0.38$ was designed to approximately match $\tau_{0}$ and $\sigma$ of $F$ s 39 . As such, all three grids represent comparable initial conditions in one or more parameters. The grids are shown in Fig. 1.

Measurements were conducted downstream of the grids in a $1.2 \mathrm{~m} \times 0.8 \mathrm{~m} \times$ $5.0 \mathrm{~m}$ wind tunnel using constant temperature hot-wire anemometry. To compare the three grids, measurements were conducted with a nano-scale thermal anemometry probe (NSTAP) [21] at $R e_{M}=28,500$ with a 1.23:1 secondary contraction installed $0.57 \mathrm{~m}$ downstream of the grids. A secondary contraction has been used to decrease anisotropy in the flow [3, 14], and was used here to collapse the anisotropy levels for all grids in the far-field to $u^{\prime} / v^{\prime}=1.10 \pm 0.05$. Measurements were also conducted in the wake of $F s 39$ at $R e_{M}=65,000$ with a X-wire and without the secondary contraction in order to investigate the region closer to the grid. Measurements were limited to the area downstream of the secondary contraction when it was installed. The measurements at $R e_{M}=65,000$ were conducted at a higher velocity and acquired for a longer sampling time to converge the peak of $\left\langle(\delta u)(\delta q)^{2}\right\rangle$ to within $\pm 5 \%$ using the $95 \%$ confidence interval. The sample time was decreased for the $R e_{M}=28,500$ measurements for efficiency. Downstream distance was measured relative to the advection time of the flow [3], $t=\int_{0}^{x} U(s)^{-1} \mathrm{~d} s$, where $U(x)$ is the local mean velocity at a position $x$. Hence, non-dimensional downstream position is expressed as $U_{0} t / M$. 


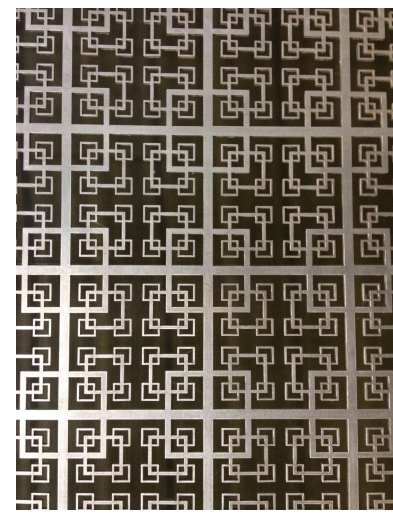

(a)

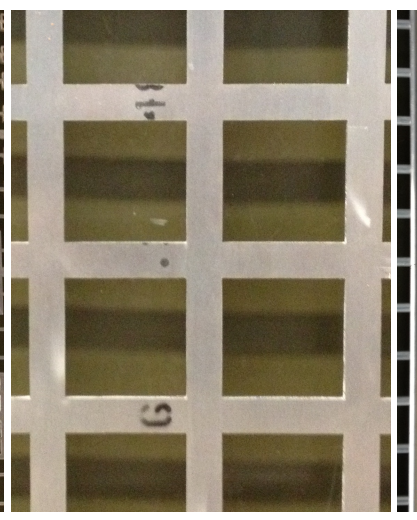

(b)

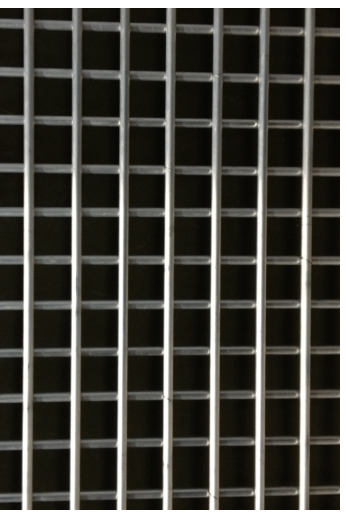

(c)

Fig. 1 Photographs of a nominally $260 \mathrm{~mm} \times 340 \mathrm{~mm}$ area of each of the $1200 \mathrm{~mm} \times 800 \mathrm{~mm}$ grids; (a) Fs39, (b) $S q 39$, (c) $R d 38$.

\section{Transition to approximate equilibrium}

The scale-by-scale kinetic energy budget for grid turbulence, (2), was assessed in normalized form given by $G / C+D / C+I / C=G^{*}+D^{*}+I^{*}=B^{*}$, where $B^{*}$ is the 'balance' of the energy budget and all variables are a function of the spatial interval $r$. When $B^{*}=1$ for all $r$, (2) is satisfied. The various terms of the scale-byscale energy budget are plotted in Fig. 2 in both the near- and far-field of $F$ s 39 at $R e_{M}=65,000$. It is immediately evident that in the far-field (2) is satisfied, while in the near-field there are significant departures from $B^{*}=1$. Contours of $B^{*}$ shown in Fig. 3 clearly identify that by $U_{0} t / M=20, B^{*} \approx 1$. Therefore, based on this analysis, the quasi-equilibrium turbulence region is $U_{0} t / M \geq 20$, and anything ahead of this may be considered non-equilibrium turbulence.

This analysis is covered in more detail for this same grid by Hearst and Lavoie [8], where they also show that there is measurable transverse transport and production in the non-equilibrium region that becomes negligible in the far-field. To confirm that the scale-by-scale energy budget results hold away from the primary axis of measurement, Hearst and Lavoie [8] also showed that the similarity form of (2) held in transverse planes in the far-field, but not in the non-equilibrium near-field.

The NSTAP measurements with a secondary contraction were designed to compare the wakes of the three grids, and were not sampled for a sufficient time to adequately resolve the terms of (2). The NSTAP measurements are more representative of the statistical analysis available for typical grid turbulence measurements. Inasmuch as this is the case, we seek a second means of assessing the transition between non-equilibrium and quasi-equilibrium turbulence that corroborates the scale-byscale kinetic energy budget results.

To address this issue, the velocity derivative skewness, $S(\partial u / \partial x)$, for all four test cases is plotted in Fig. 4. Recall that for equilibrium turbulence, $S(\partial u / \partial x)$ is con- 


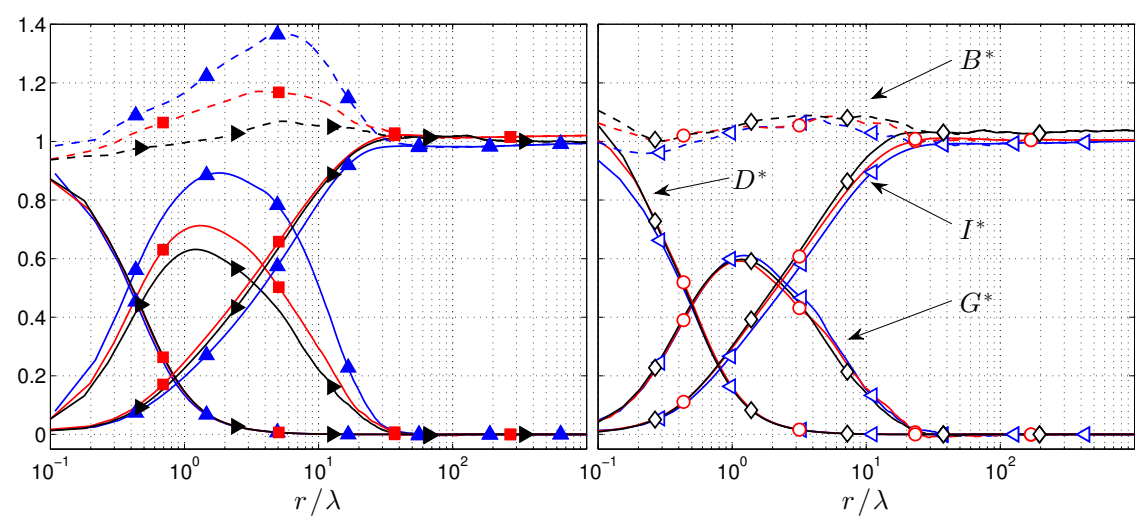

Fig. 2 The normalized scale-by-scale kinetic energy budget for grid turbulence given by (2). The left frame and filled symbols represent data acquired in the non-equilibrium region. The right frame and empty symbols represent data acquired in the quasi-equilibrium region. ( $\Delta) U_{0} t / M=10.4$, (ロ) $U_{0} t / M=15.7,(\triangleright) U_{0} t / M=18.8,(\triangleleft) U_{0} t / M=28.6$, (○) $U_{0} t / M=36.3,(\diamond) U_{0} t / M=46.1$.

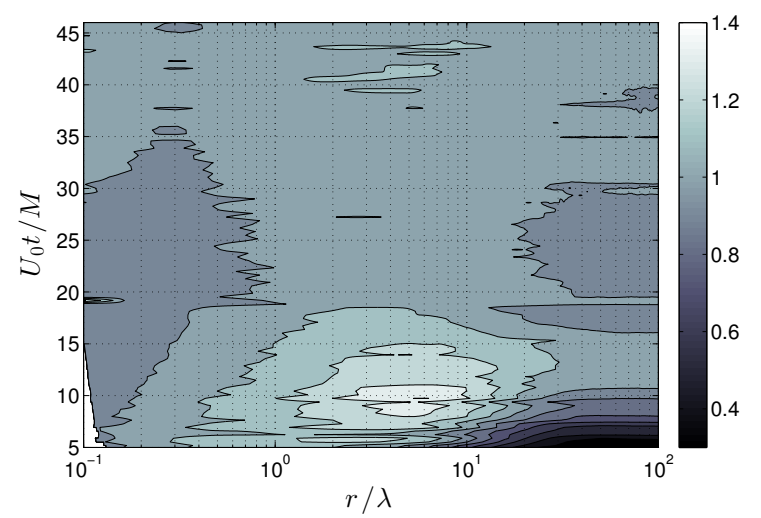

Fig. 3 Contours of the balance, $B^{*}$, of the normalized scale-by-scale kinetic energy budget for grid turbulence.

stant. From the figure, it is clear that the $F s 39$ results have an evolving $S(\partial u / \partial x)$ that becomes constant by $U_{0} t / M \approx 20$, in agreement with the scale-by-scale kinetic energy budget results. Interestingly, within the measurement range, both regular grids produce approximately constant $S(\partial u / \partial x)$, suggesting that all regular grid measurements are within the quasi-equilibrium range.

The magnitude of $S(\partial u / \partial x)$ shows a dependence on the initial conditions, as the constant far-field value is different for each test case. Sreenivasan and Antonia [17] showed that $S(\partial u / \partial x)$ has a $R e_{\lambda}$ dependence, likely related to the initial conditions, which may account for some of the differences in the far-field values. The large step change between $F s 39$ with and without the secondary contraction is likely related to the significant change in $R e_{M}$ between the experiments, and the improvement in 


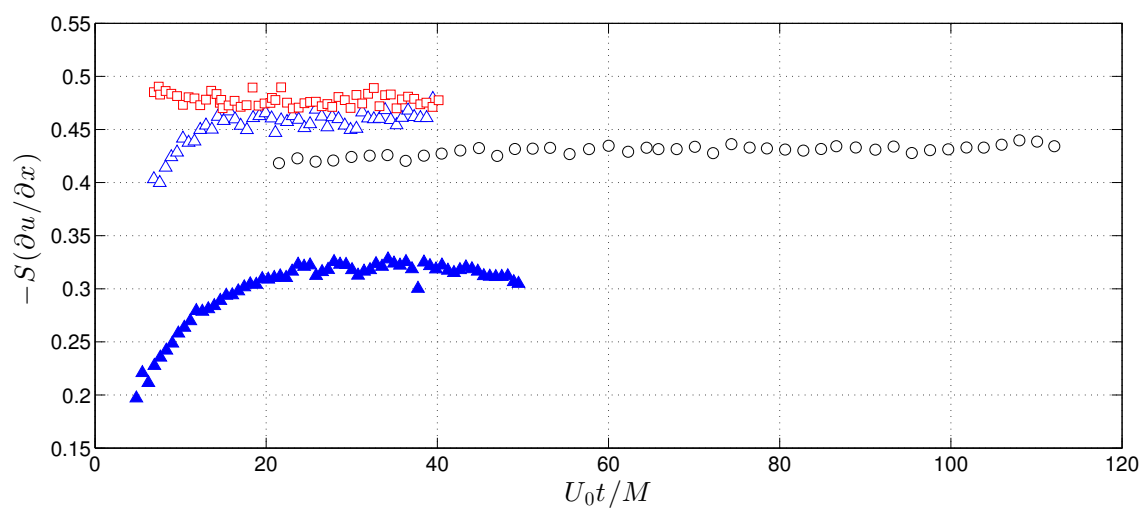

Fig. 4 Evolution of the velocity derivative skewness in the wake of $(\triangle) F s 39$, ( $\square$ ) $S q 39$, and (○) Rd38; empty symbols represent measurements with a secondary contraction, and filled symbols represent data acquired without the secondary contraction.

spatial resolution of the NSTAP for the secondary contraction case; accurate gradient estimation is highly dependent on spatial resolution [2,6]. When corrections [2] for resolution are applied to the measurements without the secondary contraction, then the far-field value becomes $\sim-0.43$, which is in better agreement with contemporary estimates of $S(\partial u / \partial x)$ in grid turbulence $[9,11]$.

\section{Reynolds number in the far-field}

The evolution of $R e_{\lambda}$ is shown in Fig. 5 for $F s 39, S q 39$, and $R d 38$ at $R e_{M}=28,500$ with a secondary contraction. Immediately apparent is that $S q 39$ produces the highest $R e_{\lambda}$ turbulence for all $U_{0} t / M$. It also appears that $R d 38$ produces higher $R e_{\lambda}$ than $F s 39$ for the $U_{0} t / M$ where they overlap. This is perhaps surprising, given that it is typically believed that fractals produce higher $R e_{\lambda}$ than regular grids $[10,19]$. The evolution of $R e_{\lambda}$ is also plotted against dimensional units in Fig. 5, and $R e_{\lambda}$ is comparable between $R d 38$ and $F s 39$ beyond $3.5 \mathrm{~m}$. Hence, regardless of the choice of normalization, in dimensional units, the $R e_{\lambda}$ produced by $R d 38$ and $F s 39$ is comparable sufficiently far downstream.

It is likely that the far-field flow of $F s 39$ has comparable $R e_{\lambda}$ to $R d 38$ because of the extended region of rapid decay experienced by the wake of $F s 39$. This is due to the longer non-equilibrium region in its wake relative to the other grids. Evidence for this hypothesis is provided in Table 1 where power-law decay exponents are estimated based on the methodology proposed in [7]. In the near-field of $F s 39$, there is a rapidly decaying region that is absent from the wake of the other two grids within the investigation range. In this extended rapidly decaying region, most of the turbulent kinetic energy injected at the turbulence generation by the grid is dissipated, leaving less energy in the far-field. This results in lower far-field $R e_{\lambda}$ 


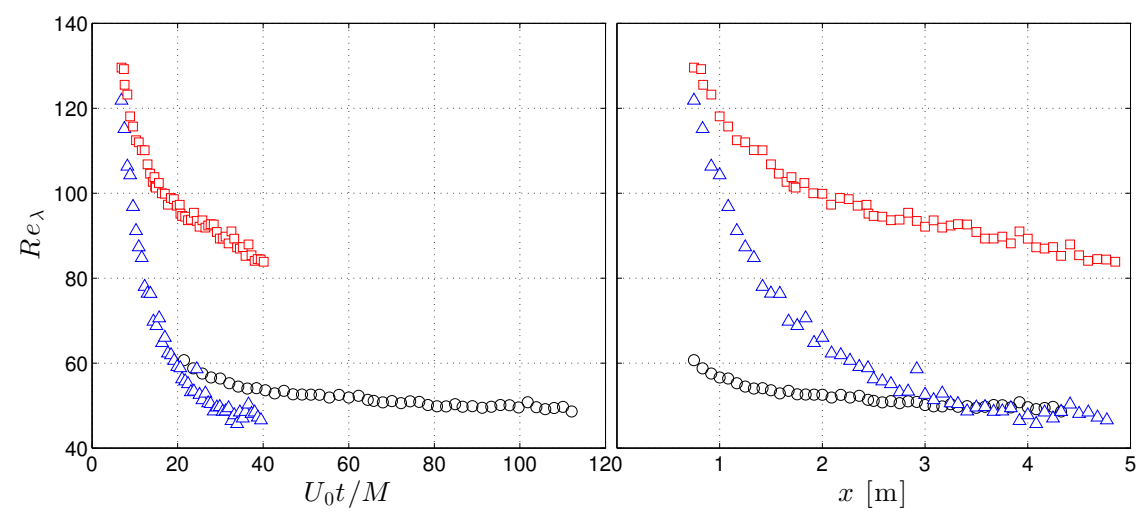

Fig. 5 Evolution of the local Reynolds number in the wake of $(\triangle) F s 39,(\square) S q 39$, and (○) Rd38. All data shown were acquired with the secondary contraction.

Table 1 Power-law decay parameters for all grids. The $\chi$ criterion is a measure of the rms difference between the measured data and the power-law fit; see [7] for details.

\begin{tabular}{lcccccc}
\hline Grid & $R e_{M}$ & Fit range $\left[U_{0} t / M\right]$ & $U_{0} t_{\min } / M$ & $U_{0} t_{0} / M$ & $n$ & $\chi[\%]$ \\
\hline$R d 38$ & 28,500 & {$[21.5,113.2]$} & 27.8 & +3.5 & -1.23 & 0.88 \\
$S q 39$ & 28,500 & {$[14.2,40.5]$} & 15.6 & +3.0 & -1.32 & 0.29 \\
$F s 39$ & 28,500 & {$[6.9,17.7]$} & 7.9 & -10.5 & -3.27 & 0.81 \\
$F s 39$ & 28,500 & {$[17.0,39.8]$} & 19.2 & +7.5 & -1.28 & 1.26 \\
\hline
\end{tabular}

than the regular grids, whose rapidly decaying non-equilibrium region is within the unobserved region closer to the grid.

\section{Conclusions}

Measurements were performed in the wakes of three turbulence generating grids with approximately the same blockage but different geometries. One grid was a square-fractal-element grid, $F s 39$, which featured an array of fractal elements mounted to a background mesh. The other two grids, $S q 39$ and $R d 38$, were regular grids with $M$ and $\tau_{0}$, respectively, matched to $F$ s39. The non-equilibrium and quasiequilibrium regions of the flow were identified by analysis of the scale-by-scale kinetic energy budget for grid turbulence and the evolution of the velocity derivative skewness. Both methodologies suggested that the flow begins to approximate equilibrium turbulence near $U_{0} t / M \approx 20$ for $F s 39$. In the far-field, it was found that $F s 39$ produced the lowest $R e_{\lambda}$ for constant $R e_{M}$. This result was verified in both non-dimensional and dimensional units, and was thus not a consequence of a chosen form of normalization. It was determined that the low far-field $R e_{\lambda}$ for $F s 39$ was a result of an extended rapid decay region in the wake of the fractal which is 
associated with the non-equilibrium region. It thus appears that while fractals may offer the benefit of high $R e_{\lambda}$ in the near-field compared to regular grids, by the time the flow has evolved into quasi-equilibrium turbulence, it has already depleted the majority of the turbulent kinetic energy injected by the turbulence generating mechanisms at the grid, resulting in a less energetic far-field.

Acknowledgements We acknowledge the financial support of the Natural Sciences and Research Council of Canada (NSERC).

\section{References}

1. Batchelor, G.K.: The theory of homogeneous turbulence. Cambridge University Press (1953)

2. Burattini, P., Lavoie, P., Antonia, R.A.: Velocity derivative skewness in isotropic turbulence and its measurement with hot wires. Exp. Fluids 45, 523-535 (2008)

3. Comte-Bellot, G., Corrsin, S.: The use of a contraction to improve the isotropy of gridgenerated turbulence. J. Fluid Mech. 25(4), 657-682 (1966)

4. Danaila, L., Anselmet, F., Antonia, R.A.: An overview of the effect of large-scale nonhomogeneities on small-scale turbulence. Phys. Fluids 14, 2475-2484 (2002)

5. Gomes-Fernandes, R., Ganapathisubramani, B., Vassilicos, J.C.: Evolution of the velocity gradient tensor in a spatially developing turbulent flow. J. Fluid Mech. 756, 252-292 (2014)

6. Hearst, R.J., Buxton, O.R.H., Ganapathisubramani, B., Lavoie, P.: Experimental estimation of fluctuating velocity and scalar gradients in turbulence. Exp. Fluids 53(4), 925-942 (2012)

7. Hearst, R.J., Lavoie, P.: Decay of turbulence generated by a square-fractal-element grid. J. Fluid Mech. 741, 567-584 (2014)

8. Hearst, R.J., Lavoie, P.: Scale-by-scale energy budget in fractal element grid-generated turbulence. J. Turb. 15(8), 540-554 (2014)

9. Hearst, R.J., Lavoie, P.: Velocity derivative skewness in fractal-generated, non-equilibrium grid turbulence. Phys. Fluids 27(7), 071,701 (2015)

10. Hurst, D., Vassilicos, J.C.: Scalings and decay of fractal-generated turbulence. Phys. Fluids 19(035103) (2007)

11. Isaza, J.C., Salazar, R., Warhaft, Z.: On grid-generated turbulence in the near- and far field regions. J. Fluid Mech. 753, 402-426 (2014)

12. Krogstad, P.A., Davidson, P.A.: Freely decaying, homogeneous turbulence generated by multiscale grids. J. Fluid Mech. 680, 417-434 (2011)

13. Krogstad, P.A., Davidson, P.A.: Near-field investigation of turbulence produced by multi-scale grids. Phys. Fluids 24(035103) (2012)

14. Lavoie, P., Djenidi, L., Antonia, R.A.: Effects of initial conditions in decaying turbulence generated by passive grids. J. Fluid Mech. 585, 395-420 (2007)

15. Mazellier, N., Vassilicos, J.C.: Turbulence without Richardson-Kolmogorov cascade. Phys. Fluids 22(075101) (2010)

16. Nagata, K., Sakai, Y., Inaba, T., Suzuki, H., Terashima, O., Suzuki, H.: Turbulence structure and turbulence kinetic energy transport in multiscale/fractal-generated turbulence. Phys. Fluids 25(065102) (2013)

17. Sreenivasan, K.R., Antonia, R.A.: The phenomenology of small-scale turbulence. Annu. Rev. Fluid Mech. 29, 435-472 (1997)

18. Valente, P.C., Vassilicos, J.C.: The decay of turbulence generated by a class of multiscale grids. J. Fluid Mech. 687, 300-340 (2011)

19. Valente, P.C., Vassilicos, J.C.: Universal dissipation scaling for nonequilibrium turbulence. Phys. Rev. Lett. 108(214503) (2012) 
20. Valente, P.C., Vassilicos, J.C.: The non-equilibrium region of grid-generated decaying turbulence. J. Fluid Mech. 744, 5-37 (2014)

21. Vallikivi, M., Hultmark, M., Bailey, S.C.C., Smits, A.J.: Turbulence measurements in pipe flow using a nano-scale thermal anemometry probe. Exp. Fluids 51, 1521-1527 (2011)

22. Zhou, Y., Nagata, K., Sakai, Y., Suzuki, H., Ito, Y., Terashima, O., Hayase, T.: Relevance of turbulence behind the single square grid to turbulence generated by regular- and multiscalegrids. Phys. Fluids 26(075105) (2014) 